\title{
Extremal problems for a family of functions with positive real part and for some related families
}

\author{
by W. JANOWSKI (Łódź)
}

1. Introduction. Denote by $\mathscr{P}(M)$ the family of all functions of the form

$$
P(z)=1+b_{1} z+b_{2} z^{2}+\ldots
$$

regular in the disk $K=\{z:|z|<1\}$ and satisfying the condition

$$
|P(z)-M|<M \quad \text { for } z \in K,
$$

where $M \geqslant 1$ is a fixed number.

Let $S^{*}(M), R(M)$ and $\delta(M)$ be the families of all functions of the form

$$
w=f(z)=z+a_{2} z^{2}+a_{3} z^{3}+\ldots
$$

regular in $K$ and satisfying the conditions

$$
\begin{aligned}
& \left|\frac{z f^{\prime}(z)}{f(z)}-M\right|<M, \quad\left|f^{\prime}(z)-M\right|<M, \\
& \left|\frac{g_{\beta}\left(e^{-i a} z\right) f(z)-e^{-i a} \cos a \cdot z}{i \sin a \cdot e^{-i a} z}-M\right|<M,
\end{aligned}
$$

where $g_{\beta}(z)=1-2 \cos \beta \cdot z+z^{2}$, sin $\alpha>0$, for $z \epsilon K$, respectively.

Next, denote by $T(M)$ and $C(M)$ the families of all functions of form (3) which are regular in $K$, real on the real axis and satisfying the conditions

$$
\left|\frac{1-z^{2}}{z} f(z)-M\right|<M, \quad\left|1-z^{2} f^{\prime}(z)-M\right|<M
$$

for $z \in K$, respectively.

In this paper, using the variational methods, the greatest lower bounds for $\operatorname{re} P(z)$ and for $\operatorname{re} \frac{z P^{\prime}(z)}{P(z)}$ within $\mathscr{P}(M)$ are given. Afterwards, the exact value of the radii of convexity for the families $S^{*}(M)$ and $R(M)$, and the radius of starlikeness for $U(M)$ are given. Moreover, the radius of convexity for the family $C(M)$ and the radius of starlikeness 
for $T(M)$ are estimated. Finally we obtain the exact bounds of $|f(z)|$ and $\left|f^{\prime}(z)\right|$ in $S^{*}(M)$ and the estimates of the coefficients in $S^{*}(M)$ and $\mathscr{P}(M)$.

2. Some properties of the families $\mathscr{P}(M), S^{*}(M), R(M), U(M)$, $T(M)$ and $C(M)$. Condition (2) means that the values of the function $P(z)$ for every $z \in K$ belong to the disk with the centre $M$ and the radius $M$.

Therefore,

$$
\operatorname{re} P(z)>0 \quad \text { for every } z \in K \text {. }
$$

The relation $\mathscr{P}\left(M_{1}\right) \subset \mathscr{P}\left(M_{2}\right)$ for $M_{1}<M_{2}$ follow easily from the definition of the class $\mathscr{P}(\boldsymbol{M})$.

Let $\mathscr{P}$ denote the family of all functions of form (1), regular in $K$ and satisfying condition (4). Then, for various values of $M, \mathscr{P}(M)$ represents a subclass of $\mathscr{P}$. Evidently $\mathscr{P}(\infty) \equiv \mathscr{P}$.

Moreover, denote by $\Omega$ the family of all functions $w(z)$ regular in $K$ and satisfying the conditions $w(0)=0$ and $|w(z)|<1$ for $z \epsilon K$. It is well known, that every function of $\mathscr{P}$ can be represented in the form

$$
p(z)=\frac{1+w(z)}{1-w(z)}
$$

where $w(z) \epsilon \Omega$. Conversely, if $w(z) \epsilon \Omega$, then the function $p(z)$ given by formula (5) belongs to $\mathscr{P}$.

If $w(z) \epsilon \Omega$, then it is easy to prove that

$$
P(z)=\frac{1+w(z)}{1-m w(z)}, \quad m=1-\frac{1}{M},
$$

is a function of $\mathscr{P}(M)$, and conversely. From (5) and (6) we conclude that

$$
P(z) \epsilon \mathscr{P}(M) \text { if and only if } p(z)=\frac{P(z)}{Q(z)} \epsilon \mathscr{P},
$$

where

(8) $\quad Q(z)=a-b P(z), \quad a=\frac{2}{1+m}, \quad b=\frac{1-m}{1+m}, \quad m=1-\frac{1}{M}$.

Using (6), we obtain easily, the estimate

$$
\frac{1-|z|}{1+m|z|} \leqslant|P(z)| \leqslant \frac{1+|z|}{1-m|z|} .
$$

The bounds are sharp, being attained by

$$
P(z)=\frac{1+\varepsilon z}{1-\varepsilon m z}, \quad|\varepsilon|=1 .
$$


Moreover, let $F$ be the family of all functions of form (3) regular in $K$. Finally, let $S^{*}$ be the subclass of $F$ which contains all functions starlike in $K$, i.e. $S^{*}$ consists of all functions mapping the disk $K$ onto domains starlike with respect to the point $w=0$.

Since any function of $F$ is starlike (and schlicht) in $K$ if and only if

$$
\mathrm{re} \frac{z f^{\prime}(z)}{f(z)}>0 \quad \text { for every } z \epsilon K,
$$

then $S^{*}(M)$ represents a subclass of $S^{*}$. Evidently $S^{*}(\infty) \equiv S^{*}$.

It is known [3] that any function $f(z)$ of $F$ belongs to the family $S^{*}$ if and only if $\frac{z f^{\prime}(z)}{f(z)} \subset \mathscr{P}$.

Let $R$ by the subclass of all functions of $F$ whose first derivative have positive real part in $K$. Then $R(M) \subset R$ and $R(\infty) \equiv R$.

It is known [5] that $R$ is a subset of $S$, where $S \subset F$ is the class that consists of all functions schlicht in $K$.

Moreover, let $U \subset F$ be the class that consists of all functions $f(z)$ starlike in the direction of the real axis, i.e. for any $r, 0<r<1$, and $f(z) \in U$ the image of the circle $|z|=r$ under $f(z)$ meets the real axis not more than at two points [6]. On the other hand, a function of $F$ belongs to $U$ if and only if

$$
P(z)=\frac{g_{\beta}\left(e^{-i a} z\right) f(z)-e^{-i a} \cos \alpha \cdot z}{i \sin \alpha \cdot e^{-i a} z},
$$

where $g_{\beta}(z)=1-2 \cos \beta \cdot z+z^{2}, \sin \alpha>0$, is a function of $\mathscr{P},[6]$.

Hence $U(M) \subset U$ and $U(\infty) \equiv U$.

Next, denote by $T$ the subclass of all functions of $F$ which are typically-real in $K$, i.e. they assume real values if and only if $z$ is real [9]. Since a function of $F$ is typically-real in $K$ if and only if

$$
P(z)=\frac{1-z^{2}}{z} f(z)
$$

is a function of $\mathscr{P}$ with real coefficients [9], then $T(M) \subset T$ and $T(\infty) \equiv T$. Evidently $T$ is a subclass of $U$ and $T(M)$ is a subclass of $U(M)$.

Finally, let $C$ be the family of all functions $f(z) \in F$ which are real on the real axis and map $K$ onto a domain convex in the direction of the imaginary axis, i.e. for any $r, 0<r<1$, and $f(z) \in C$ the image of the circle $|z|=r$ under $f(z)$ meets every straight line parallel to the imaginary axis not more than at two points [7]. Since a function of $F$ belongs to $C$ if and only if $z f^{\prime}(z)$ is typically-real [7], then

$$
P(z)=\left(1-z^{2}\right) f^{\prime}(z)
$$

is a function of positive real part with real coefficients.

Therefore $C(\boldsymbol{M})$ represents a subclass of $C$. Evidently $C(\infty) \equiv C$. 
3. A variational formula for the class $\mathscr{P}(M)$. If $p(z) \epsilon \mathscr{P}$, then [8] so is

$$
p_{s}(z)=p(z)-\varepsilon\left(1-\left|z_{0}\right|^{2}\right) z s(z)+o(\varepsilon),
$$

where

$$
s(z)=\left(\frac{\eta z}{z_{0}\left(z-z_{0}\right)}+\frac{\bar{\eta} z}{1-\bar{z}_{0} z}-\frac{\eta p(z)}{p\left(z_{0}\right)\left(z-z_{0}\right)}+\frac{\bar{\eta} z p(z)}{\overline{p\left(z_{0}\right)}\left(1-\bar{z}_{0} z\right)}\right)_{z}^{\prime},
$$

$z_{0} \in K,|\eta|=1, \varepsilon>0$ and the error term $o(\varepsilon)$ is an analytic function in $z$ uniformly bounded in each interior region of $K$.

Since there is a one-one correspondence given by (7) and (8) between the function $p(z)$ of $\mathscr{P}$ and the function $P(z)$ of $\mathscr{P}(M)$, we obtain easily, after some calculation, the following variational formula within $\mathscr{P}(M)$ :

$$
P_{\varepsilon}(z)=P(z)-\varepsilon\left(1-\left|z_{0}\right|^{2}\right) z \mathcal{S}(z) Q^{2}(z)+o(\varepsilon),
$$

where

$$
\begin{aligned}
S(z)= & \frac{\eta}{z_{0}\left(z_{0}-z\right)^{2}}\left[z_{0} \frac{P(z)}{Q(z)} \frac{Q\left(z_{0}\right)}{P\left(z_{0}\right)}-z\right]+ \\
& +\frac{\eta}{z_{0}\left(z_{0}-z\right)}\left[z_{0} \frac{a P^{\prime}(z)}{Q^{2}(z)} \frac{Q\left(z_{0}\right)}{P\left(z_{0}\right)}-1\right]+ \\
& +\frac{\bar{\eta}}{\left(1-z_{0} z\right)^{2}}\left[1+\frac{P(z)}{Q(z)} \frac{\overline{Q\left(z_{0}\right)}}{\overline{P\left(z_{0}\right)}}+\frac{\bar{\eta} z}{1-\bar{z}_{0} z} \frac{a P^{\prime}(z)}{\overline{Q^{2}(z)}} \frac{\overline{Q\left(z_{0}\right)}}{\overline{P\left(z_{0}\right)}}\right]
\end{aligned}
$$
4. Extremal problems for $\operatorname{re} P(z)$ and $\operatorname{re} \frac{z p^{\prime}(z)}{P(z)}$ within the family
$\mathscr{P}(M)$. It is well known that

$$
\inf _{p e \theta} \operatorname{rep}(z)=\frac{1-r}{1+r}, \quad|z|=r<1 \text {. }
$$

THEOREM 1. For every $P(z) \in \mathscr{P}(M)$ and $|z|=r, 0<r<1$, we have

$$
\operatorname{re} P(z) \geqslant \frac{1-r}{1+m r}
$$

The bound is sharp, being attained by

$$
P(z)=\frac{1-\varepsilon z}{1+\varepsilon m z}, \quad|\varepsilon|=1
$$

Proof. Let $P(z)$ be the minimizing function for $\operatorname{re} P(z), z \in K$ being fixed and $P_{s}(z)$ belonging to $\mathscr{P}(M)$. Now we may suppose without loss of generality that the minimum is attained on the real axis at $z=r>0$ since $P_{t}(z)=P\left(e^{i t} z\right)$ belongs to the family $\mathscr{P}(M)$ for $t$ real. Applying the variational formula (9) we obtain 


$$
\begin{gathered}
\frac{\mathrm{re}\left[P_{\varepsilon}(r)-P(r)\right]}{1-\left|z_{0}\right|^{2}}=-\varepsilon Q^{2}(r)\left\{\frac{\eta r}{z_{0}\left(z_{0}-r\right)^{2}}\left[z_{0} \frac{P(r)}{Q(r)} \frac{Q\left(z_{0}\right)}{P\left(z_{0}\right)}-r\right]+\right. \\
+\frac{\eta r}{z_{0}\left(z_{0}-r\right)}\left[z_{0} \frac{a P^{\prime}(r)}{Q^{2}(r)} \frac{Q\left(z_{0}\right)}{P\left(z_{0}\right)}-1\right]+\frac{\overline{\eta r}}{\left(1-\bar{z}_{0} r\right)^{2}}\left[1+\frac{P(r)}{Q(r)} \frac{\overline{Q\left(z_{0}\right)}}{\overline{P\left(z_{0}\right)}}\right]+ \\
\left.+\frac{\overline{\eta r^{2}}}{1-\bar{z}_{0} r} \frac{a P^{\prime}(r)}{Q^{2}(r)} \frac{\overline{Q\left(z_{0}\right)}}{\overline{P\left(z_{0}\right)}}\right\}+o(\varepsilon) \geqslant 0
\end{gathered}
$$

Using the fact the re $x=$ re $\bar{x}$ for any complex $x$, and replacing $z_{0}$ by $z$ in (13), we obtain $\operatorname{re} \eta H \leqslant 0$ for all $\eta,|\eta|=1$, where

$$
\begin{aligned}
H=H(r)=\frac{Q^{2}(r)}{z(z-r)^{2}} & {\left[z \frac{P(r)}{Q(r)} \frac{Q(z)}{P(z)}-r\right]+\frac{Q^{2}(r)}{z(z-r)}\left[z \frac{a P^{\prime}(r)}{Q^{2}(r)} \frac{Q(z)}{P(z)}-1\right]+} \\
+ & \frac{\overline{Q^{2}(r)}}{(1-r z)^{2}}\left[1+\frac{\overline{P(r)}}{\overline{Q(r)}} \frac{Q(z)}{P(z)}\right]+\frac{r \overline{Q^{2}(r)}}{1-r z} \frac{a \overline{P^{\prime}(r)}}{\overline{Q^{2}(r)}} \frac{Q(z)}{P(z)} .
\end{aligned}
$$

Since $\eta,|\eta|=1$, is arbitrary, it follows that $H=0$. Now, for real values of $t$ we have

$$
P_{\ell}(r)=P\left(e^{i t} r\right)=P(r)+i t r P^{\prime}(r)+o(t) .
$$

Therefore

$$
\mathbf{r e}\left(P_{t}(r)-P(r)\right)=-\operatorname{trim} P^{\prime}(r)+o(t) \geqslant 0 .
$$

Since $t$ is an arbitrary real number, then we obtain $\operatorname{im} P^{\prime}(r)=0$. Hence the equation $H=0$ can be written in the form

$$
\begin{aligned}
& Q^{2}(r)\left[\frac{1}{(z-r)^{2}} \frac{P(r)}{Q(r)} \frac{Q(z)}{P(z)}+\frac{a}{z-r} \frac{P^{\prime}(r)}{Q^{2}(r)} \frac{Q(z)}{P(z)}-\frac{1}{z-r^{2}}\right]+ \\
+ & \overline{Q^{2}(r)}\left[\frac{1}{(1-r z)^{2}} \frac{\overline{P(r)}}{\overline{Q(r)}} \frac{Q(z)}{P(z)}+\frac{a r}{1-r z} \frac{P^{\prime}(r)}{\overline{Q^{2}(r)}} \frac{Q(z)}{P(z)}+\frac{1}{(1-r z)^{2}}\right]=0 .
\end{aligned}
$$

By $P(0)=1$ and (14) we have

$$
\frac{a\left(1-r^{2}\right)}{r} P^{\prime}(r)=\frac{Q(r)[P(r)-Q(r)]}{r^{2}}+\overline{Q(r)}[\overline{P(r)}+\overline{Q(r)}] .
$$

Since $P^{\prime}(r)$ is real, then, by (15), we have

$$
\begin{aligned}
\frac{Q(r)[P(r)-Q(r)]}{r^{2}}+\overline{Q(r)} & {[\overline{P(r)}+\overline{Q(r)}] } \\
= & \frac{\overline{Q(r)}[\overline{P(r)}-\overline{Q(r)}]}{r^{2}}+Q(r)[P(r)+Q(r)] .
\end{aligned}
$$


Therefore

$$
\begin{aligned}
{[P(r)-\overline{P(r)}]\left[a\left(1-r^{2}\right)\right.} & +2 a b\left(1+r^{2}\right)- \\
& \left.-\left(b\left(1-r^{2}\right)+b^{2}\left(1+r^{2}\right)\right)(P(r)+\overline{P(r)})\right]=0 .
\end{aligned}
$$

It can easily be verified that, by $P(z) \in \mathscr{P}(M)$, the second factor in (16) does not vanish. Consequently

$$
P(r)=\overline{P(r)} \text {. }
$$

Thus equation (14) takes the form

$$
\begin{aligned}
(1-r z)^{2} & {\left[\frac{P(r)}{Q(r)} \frac{Q(z)}{P(z)}+\frac{a P^{\prime}(r)(z-r)}{Q^{2}(r)} \frac{Q(z)}{P(z)}-1\right]+} \\
& +(z-r)^{2}\left[\frac{P(r)}{Q(r)} \frac{Q(z)}{P(z)}+\frac{a r P^{\prime}(r)(1-r z)}{Q^{2}(r)} \frac{Q(z)}{P(z)}+1\right]=0 .
\end{aligned}
$$

Solving this equation with respect to $Q(z) / P(z)$ we obtain

$$
\left(K_{0} z^{2}+K_{1} z+K_{2}\right) \frac{Q(z)}{P(z)}=\left(1-r^{2}\right)\left(1-z^{2}\right),
$$

where

$$
\begin{aligned}
& K_{0}=\left(1+r^{2}\right) \frac{P(r)}{Q(r)}-a r\left(1-r^{2}\right) \frac{P^{\prime}(r)}{Q^{2}(r)}, \\
& K_{1}=-4 r \frac{P(r)}{Q(r)}+a\left(1-r^{4}\right) \frac{P^{\prime}(r)}{Q^{2}(r)}, \\
& K_{2}=K_{0} .
\end{aligned}
$$

By (17) and (15) we have

Hence

$$
\frac{a P^{\prime}(r)}{Q^{2}(r)}=\frac{1+r^{2}}{r\left(1-r^{2}\right)} \frac{P(r)}{Q(r)}-\frac{1}{r} .
$$

and (18) becomes

$$
\begin{aligned}
& K_{0}=1-r^{2} \\
& K_{1}=\frac{1-r^{2}}{r}\left[\left(1-r^{2}\right) \frac{P(r)}{Q(r)}-\left(1+r^{2}\right)\right]
\end{aligned}
$$

where

$$
\left(z^{2}+A z+1\right) \frac{Q(z)}{P(z)}=1-z^{2}
$$

$$
A=\frac{1-r^{2}}{r} \frac{P(r)}{Q(r)}-\frac{1+r^{2}}{r}
$$

Solving this equation with respect to $P(z)$ we get

$$
P(z)=\frac{(1-z)^{2}+(2+A) z}{(1-z)(1+m z)+\frac{1}{2}(1+m)(2+A) z} .
$$


By $P(r)>0$ we have $A \geqslant-2$. Since the minimum of the real part of functions belonging to $\mathscr{P}(M)$ is attained for $P(r)$, we must take $A=-2$. In this case

$$
P(z)=\frac{1-z}{1+m z} .
$$

Hence (11) follows with equality for function (12) only. Passing to the limit in (11) as $m \rightarrow 1$ we obtain the corresponding result for the functions of the class $\mathscr{P}$.

THEOREM 2. For every $P(z) \in \mathscr{P}(M)$ and $|z|=r, 0<r<1$, we have

$$
\text { re } \frac{z P^{\prime}(z)}{P(z)} \geqslant \frac{-(1+m) r}{(1-r)(1+m r)} .
$$

The bound is sharp, being attained by

$$
P(z)=\frac{1-\varepsilon z}{1+\varepsilon m z}, \quad|\varepsilon|=1 .
$$

Proof. Let $P(z)$ be the minimizing function for $r e \frac{z P^{\prime}(z)}{P(z)}, z \in K$ being fixed and $P(z)$ belonging to $\mathscr{P}(M)$. Now we may suppose in analogy to the previous theorem that the minimum is attained on the real axis at $z=r>0$.

Using the variational formula (9) we obtain

$$
\begin{aligned}
\frac{\mathrm{re}\left(\frac{r P_{e}^{\prime}(r)}{P_{e}(r)}-\frac{r P^{\prime}(r)}{P(r)}\right)}{1-\left|z_{0}\right|^{2}}= & -\varepsilon r \frac{Q(r)}{P(r)}\left\{r \left[Q(r) S^{\prime}(r)+2 S(r) Q^{\prime}(r)-\right.\right. \\
& \left.\left.-\frac{Q(r)}{P(r)} S(r) P^{\prime}(r)\right]-S(r) Q(r)\right\}+o(\varepsilon) \geqslant 0,
\end{aligned}
$$

where $S(z)$ is given by formula (10).

After some simplification we obtain

$$
\begin{aligned}
\frac{1}{1-\left|z_{0}\right|^{2}} \mathrm{re}\left(\frac{r P_{a}^{\prime}(r)}{P_{e}(r)}-\frac{r P^{\prime}(r)}{P(r)}\right) \\
=-\varepsilon r \frac{Q(r)}{P(r)}\left[A(r) S(r)+r Q(r) S^{\prime}(r)\right]+o(\varepsilon) \geqslant 0,
\end{aligned}
$$

where

$$
A(r)=Q(r)\left(1-\frac{r P^{\prime}(r)}{P(r)}\right)-2 r b P^{\prime}(r) .
$$

Next we differentiale function (10) with respect to $z$ and apply to (21) the expressions for $S(r)$ and $S^{\prime}(r)$ given by (10). Using the fact that 
re $x=\mathrm{r} \Theta \bar{x}$ for any complex $x$, and replacing $z_{0}$ by $z$ in (21), we obtain re $\eta r H \leqslant 0$ for all $\eta,|\eta|=1$, where

$$
\begin{aligned}
& H=H(r)=\frac{Q(r) A(r)}{P(r)}\left\{\frac{1}{z(z-r)^{2}}\left[z \frac{P(r)}{Q(r)} \frac{Q(z)}{P(z)}-r\right]+\right. \\
& \left.+\frac{1}{z(z-r)}\left[a z \frac{P^{\prime}(r)}{Q^{2}(r)} \frac{Q(z)}{P(z)}-1\right]\right\}+\frac{r Q^{2}(r)}{P(r)}\left\{\frac{2}{z(z-r)^{3}}\left[z \frac{P(r)}{Q(r)} \frac{Q(z)}{P(z)}-r\right]+\right. \\
& +\frac{1}{z(z-r)^{2}}\left[a z \frac{P^{\prime}(r)}{Q^{2}(r)} \frac{Q(z)}{P(z)}+z\left(\frac{P(r)}{Q(r)}\right)_{r}^{\prime} \frac{Q(z)}{P(z)}-2\right]+ \\
& \left.+\frac{a}{z-r}\left(\frac{P^{\prime}(r)}{Q^{2}(r)}\right)_{r}^{\prime} \frac{Q(z)}{P(z)}\right\}+\frac{\overline{Q(r)} \overline{A(r)}}{\overline{P(r)}}\left\{\frac{1}{(1-r z)^{2}}\left[\frac{P(r)}{Q(r)} \frac{Q(z)}{P(z)}+1\right]+\right. \\
& \left.+\frac{a r}{1-r z} \frac{\overline{P^{\prime}(r)}}{\overline{Q^{2}(r)}} \frac{Q(z)}{P(z)}\right\}+\frac{r \overline{Q^{2}(r)}}{\overline{P(r)}}\left\{\overline{(1-r z)^{3}}\left[\frac{2 z(r)}{\overline{Q(r)}} \overline{P(z)}+1\right]+\right. \\
& \left.+\frac{1}{(1-r z)^{2}}\left[\frac{\overline{P^{\prime}(r)}}{\overline{Q^{2}(r)}}+\left(\frac{\overline{P(r)}}{Q(r)}\right)_{r}^{\prime}\right] \frac{Q(z)}{P(z)}+\frac{a r}{1-r z}\left(\frac{\overline{P^{\prime}(r)}}{Q^{2}(r)}\right)_{r}^{\prime} \frac{Q(z)}{P(z)}\right\} .
\end{aligned}
$$

Since $\eta,|\eta|=1$, is arbitrary, it follows that $H=0$. Now, let

$$
\begin{gathered}
B=\left[\frac{r P^{\prime}(r)}{P(r)}+1\right] Q(r), \quad C=a\left[\frac{P^{\prime}(r)}{P(r)}-\frac{r P^{\prime 2}(r)}{P^{2}(r)}+\frac{r P^{\prime \prime}(r)}{P(r)}\right], \\
D=2 r Q(r), \quad E=\frac{Q(r)}{P(r)} A(r), \quad F=\frac{r Q^{2}(r)}{P(r)} .
\end{gathered}
$$

Then the equation $H=0$ after some calculation becomes

$$
\begin{aligned}
\{[D+B(z-r) & \left.+C(z-r)^{2}\right](1-r z)^{3}+ \\
& \left.+\left[\bar{D}+\bar{B}(1-r z)+r \bar{C}(1-r z)^{2}\right]\right\} \frac{Q(z)}{P(z)} \\
= & {[2 F+E(z-r)](1-r z)^{3}-[2 \bar{F} z+\bar{E}(1-r z)](z-r)^{3} . }
\end{aligned}
$$

Wo claim that

$$
C=\bar{C}
$$

In fact, for real $t$ we have

$$
\mathrm{re}\left[\frac{r P_{t}^{\prime}(r)}{P_{t}(r)}-\frac{r P^{\prime}(r)}{P(r)}\right]=\mathrm{re} \frac{r}{a} i t C+o(t) \geqslant 0 .
$$

Since $t$ is an arbitrary number, we obtain

$$
\operatorname{im} C=0 .
$$


Solving equation (22) with respect to $\frac{P(z)}{Q(z)}$, we get

$$
\frac{P(z)}{Q(z)}=\frac{p_{0}+p_{1} z+p_{2} z^{2}+\bar{p}_{1} z^{3}+\bar{p}_{0} z^{4}}{q_{0}+q_{1} z+q_{2} z^{2}-\bar{q}_{1} z^{3}-\bar{q}_{0} z^{4}},
$$

where

$$
\begin{aligned}
& p_{0}=D+r^{2}\left(1-r^{2}\right) C-r B-r^{3} \bar{B} \\
& p_{1}=\left(1+3 r^{2}\right) B+r^{2}\left(3+r^{2}\right) \bar{B}+2 r\left(r^{4}-1\right) C-3 r D-r^{3} \bar{D} \\
& p_{2}=\left(1+3 r^{2}-3 r^{4}-r^{6}\right) C+3 r^{2}(D+\bar{D})-3 r\left(1+r^{2}\right)(B+\bar{B}), \\
& q_{0}=2 F-r E+r^{3} \bar{E} \\
& q_{1}=\left(3 r^{2}+1\right) E+2 \bar{F} r^{3}-r^{2}\left(3+r^{2}\right) \bar{E}-6 r F \\
& q_{2}=6 r^{2}(F-\bar{F})-3 r\left(1+r^{2}\right)(E-\bar{E}) .
\end{aligned}
$$

Applying the results contained in [2] we obtain that the function $\frac{P(z)}{Q(z)}$ can be expressed in the form

$$
\frac{P(z)}{Q(z)}=\sum_{k=1}^{4} \lambda_{k} \frac{1+\varepsilon_{k} z}{1-\varepsilon_{k} z}
$$

where

$$
0 \leqslant \lambda_{k} \leqslant 1, \quad \sum_{k=1}^{4} \lambda_{k}=1, \quad\left|\varepsilon_{k}\right|=1 \quad \text { for } k=1,2,3,4 .
$$

Now, differentiating function (24) with respect to $z$, using (8), and substituting $r$ for $z=|z|$, we obtain

$$
\frac{a P^{\prime}(r)}{Q^{2}(r)}=\sum_{k=1}^{4} \frac{2 \lambda_{k} \varepsilon_{k}}{\left(1-\varepsilon_{k} z\right)^{2}}
$$

Therefore

$$
a\left|\frac{P^{\prime}(r)}{Q^{2}(r)}\right| \leqslant 2 \sum_{k=1}^{4} \frac{\lambda_{k}}{\left|1-\varepsilon_{k} r\right|^{2}}
$$

Since

$$
\mathrm{re} \frac{P(r)}{Q(r)}=\left(1-r^{2}\right) \sum_{k=1}^{4} \frac{\lambda_{k}}{\left|1-\varepsilon_{k} r\right|^{2}}
$$

we obtain

$$
a\left|\frac{P^{\prime}(r)}{Q^{2}(r)}\right| \leqslant \frac{2}{1-r^{2}} \text { re } \frac{P(r)}{Q(r)}
$$


Hence

$$
\left.\mid r P^{\prime}(r)\right\} \leqslant \frac{2 r}{a\left(1-r^{2}\right)} \operatorname{re} P(r) \overline{Q(r)} .
$$

Next, by (8), we have

$$
\operatorname{re} P(r) \overline{Q(r)}=a \operatorname{re} P(r)-b|P(r)|^{2} \leqslant(a-b)|P(r)| \mathrm{re} P(r)
$$

and consequently by

$$
|P(z)| \geqslant \frac{1-r}{1+m r}, \quad|z|=r,
$$

we obtain

$$
\left|r P^{\prime}(r)\right| \leqslant \frac{2 r}{a\left(1-r^{2}\right)}\left(a-b \frac{1-r}{1+m r}\right) \operatorname{re} P(r)=\frac{(1+m) r}{(1-r)(1+m r)} \operatorname{re} P(r)
$$

and

$$
\frac{\left|r P^{\prime}(r)\right|}{r \theta P(r)} \leqslant \frac{(1+m) r}{(1-r)(1+m r)}
$$

Since

we obtain finally

$$
\mathrm{re} \frac{r P^{\prime}(r)}{P(r)} \geqslant-\left|\frac{r P^{\prime}(r)}{P(r)}\right| \geqslant-\frac{\left|r P^{\prime}(r)\right|}{\operatorname{reP}(r)},
$$

$$
\mathrm{re} \frac{r P^{\prime}(r)}{P(r)} \geqslant-\frac{(1+m) r}{(1-r)(1+m r)},
$$

i.e. inequality (19). Since equality holds in (25)-(29) if and only if $\varepsilon_{k}=1$, $k=1,2,3,4$, then the extremal function is given by formula (20), and this completes the proof.

Passing to the limit in (19) as $m \rightarrow 1$ we obtain a result of Libera [2].

COROLlaRY. For every $P(z) \in \mathscr{P}(M)$ and $|z|=r, 0<r<1$, we have

$$
\text { re } \frac{z P^{\prime}(z)}{P(z)} \leqslant \frac{(1+m) r}{(1-r)(1+m r)}
$$

with equality if and only if

$$
P(z)=\frac{1-\varepsilon z}{1+\varepsilon m z}, \quad|\varepsilon|=1 \text {. }
$$

5. The radii of convexity for the families $S(M)$ and $R(M)$. The radius of starlikeness of $U(M)$.

THEOREM 3. The radius of convexity of the family $\mathcal{S}^{*}(M)$ is givon by the formula

$$
\varrho=\frac{3+m-\sqrt{m^{2}+6 m+5}}{2}
$$


Proof. If $f(z) \in \mathcal{S}^{*}(M)$, then

$$
\frac{z f^{\prime}(z)}{f(z)}=P(z)
$$

for some function $P(z) \in \mathscr{P}(M)$. Differentiating (31) we obtain

$$
\frac{f^{\prime}(z)}{f(z)}+\frac{z f^{\prime \prime}(z)}{f^{\prime}(z)}-z\left(\frac{f^{\prime}(z)}{f(z)}\right)^{2}=P^{\prime}(z) \text {. }
$$

An easy calculation yields

Thus

$$
1+\frac{z f^{\prime \prime}(z)}{f^{\prime}(z)}=P(z)+\frac{z P^{\prime}(z)}{P(z)} .
$$

$$
\mathrm{re}\left(1+\frac{z f^{\prime \prime}(z)}{f^{\prime}(z)}\right) \geqslant \min _{P(z) \in \mathscr{P}(M)} \operatorname{re} P(z)-\max _{P(z) \in \mathscr{P}^{\prime}(M)}\left|\frac{z P^{\prime}(z)}{P(z)}\right|, \quad|z|=r .
$$

By (11) and

$$
\left|\frac{z P^{\prime}(z)}{P(z)}\right| \leqslant \frac{(1+m) r}{(1-r)(1+m r)}, \quad|z|=r, 0 \leqslant r<1,
$$

we get

$$
\mathrm{re}\left(1+\frac{z f^{\prime \prime}(z)}{f^{\prime}(z)}\right) \geqslant \frac{r^{2}-(3+m) r+1}{(1-r)(1+m r)} .
$$

The denominator of the expression on the right-hand side of inequality (33) is positive for $0 \leqslant r<1$. Thus the inequality

$$
\mathrm{re}\left(1+\frac{z f^{\prime \prime}(z)}{f^{\prime}(z)}\right)>0
$$

is valid for $r=|z|<\varrho$, where $\varrho$ is given by formula (30). Hence the radius of convexity $R_{c}$ for $S^{*}(M)$ is not less than $\varrho$.

The function

$$
f^{*}(z)=\frac{z}{(1+\varepsilon m z)^{(1+m) / m}}, \quad|\varepsilon|=1, m \neq 0,
$$

as it can easily be verified, belong to $\mathcal{S}^{*}(M)$, where $M \neq 1$.

Since

$$
1+\frac{z f^{* \prime \prime}(z)}{f^{* \prime}(z)}=\frac{\varepsilon^{2} z^{2}-\varepsilon(3+m) z+1}{(1-\varepsilon z)(1+\varepsilon m z)}
$$

we get

$$
\mathrm{r \theta}\left(1+\frac{z f^{* \prime \prime}(z)}{f^{* \prime}(z)}\right)=0
$$

for $z=\bar{\varepsilon} \varrho$. Thus the function $f^{*}(z)$ is not convex in the disk $|z|<r$ for $r>\varrho$. Hence, by $R_{c} \geqslant \varrho$ we obtain $R_{c}=\varrho$ in case where $m \neq 0$. 
The function

$$
f^{* *}(z)=z e^{\varepsilon z}, \quad|\varepsilon|=1,
$$

belongs to $S^{*}(1)$. Since

we get

$$
1+\frac{z f^{* * \prime \prime}(z)}{f^{* * \prime}(z)}=\frac{\varepsilon^{2} z^{2}+3 \varepsilon z+1}{1+\varepsilon z}
$$

$$
\mathrm{re}\left(1+\frac{z f^{* * \prime \prime}(z)}{f^{* * \prime \prime}(z)}\right)=0
$$

for $z=-\bar{\varepsilon} \varrho$. Thus, if $m=0$ we obtain likewise $R_{c}=\varrho$, and this completes the proof.

Passing to the limit in (30) as $m \rightarrow 1$ we obtain $\varrho=2-\sqrt{3},[4]$.

Similarly, we obtain

Theorem 4. The radius of convexity for the family $R(M)$ is equal to

$$
\varrho= \begin{cases}(\sqrt{1+m}-1) / m, & \text { if } m \neq 0 \\ \frac{1}{2}, & \text { if } m=0 .\end{cases}
$$

The functions

$$
f^{*}(z)=\bar{\varepsilon} \frac{m+1}{m^{2}} \log (1+\varepsilon m z)-\frac{z}{m}, \quad|\varepsilon|=1, \log 1=0
$$

and

$$
f^{* *}(z)=z-\frac{1}{2} \varepsilon z^{2}, \quad|\varepsilon|=1
$$

from $R(M)(M \neq 1)$ and from $R(1)$, respectively, shows that this result is sharp. Passing to the limit in (36) as $m \rightarrow 1$, we obtain $\varrho=\sqrt{2}-1$, [2].

THEOREM 5. The radius of starlikeness for the family $U(M)$ is equal to

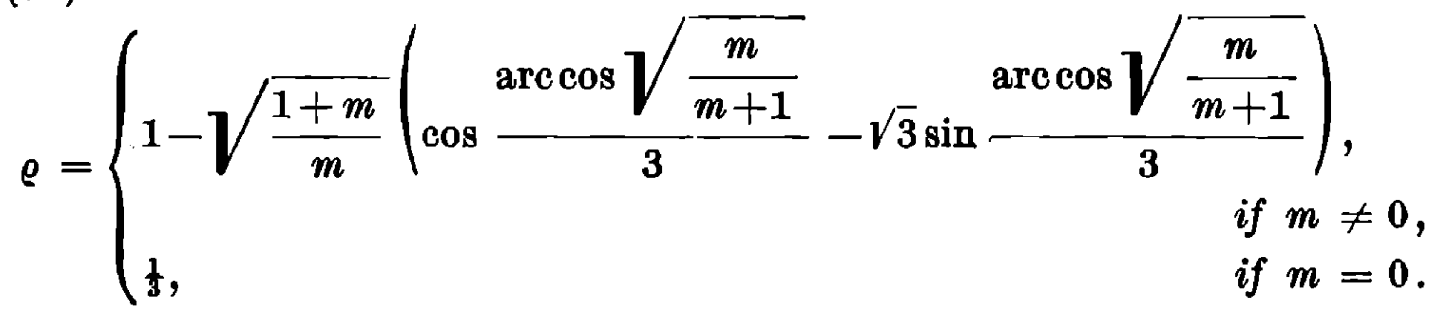

Proof. If $f(z) \in U(M)$, then

$$
\frac{g_{\beta}\left(e^{-i a} z\right) f(z)-e^{-i a} \cos \alpha \cdot z}{i \sin a \cdot e^{-i a} z}=P(z)
$$

for some function $P(z) \in \mathscr{P}(M)$. Differentiating (40) with respect to $z$ and simplifying the result we get

$$
\frac{z f^{\prime}(z)}{f(z)}=\frac{1-e^{-2 i \alpha} z^{2}}{1-2 e^{-i a} \cos \beta \cdot z+e^{-2 i a} z^{2}}+\frac{z P^{\prime}(z)}{P(z)+h}, \quad h=-i \cot a .
$$


Thus

re $\frac{z f^{\prime}(z)}{f(z)} \geqslant \min _{a, \beta} r e \frac{1-e^{-2 i a} z^{2}}{1-2 e^{-i a} \cos \beta \cdot z+e^{-2 i a} z^{2}}-\max _{P(z) \in \mathscr{P}(M)}\left|\frac{z P^{\prime}(z)}{P(z)+h}\right|, \quad|z|=r$.

Let $z=r e^{i \varphi}, 0 \leqslant \varphi<2 \pi$, and

$$
A(\alpha, \beta, \varphi)=\operatorname{re} \frac{1-e^{2 i(\varphi-a)} r^{2}}{1-2 e^{i(\varphi-a)} \cos \beta \cdot r+e^{2 i(\Phi-a)} \cdot r^{2}} .
$$

Since

$$
\min _{a, \beta, \Phi} A(a, \beta, \varphi)=A\left(\frac{\pi}{2}, 0,-\frac{\pi}{2}\right),
$$

then

$$
\mathrm{re} \frac{z f^{\prime}(z)}{f(z)} \geqslant \frac{1-r}{1+r}-\max _{P(z) \in \mathcal{P}_{(M)}}\left|\frac{z P^{\prime}(z)}{P(z)+h}\right| .
$$

Next, we have

$$
\left|\frac{z P^{\prime}(z)}{P(z)+h}\right| \leqslant \frac{z P^{\prime}(z)}{\operatorname{reP}(z)}
$$

and, by (28), we obtain

$$
\left|\frac{z P^{\prime}(z)}{P(z)+h}\right| \leqslant-\frac{(1+m) r}{(1-r)} \frac{(1+m r)}{.}
$$

Therefore

$$
\mathrm{re} \frac{z f^{\prime}(z)}{f(z)} \geqslant \frac{1-r}{1+r}-\frac{(1+m) r}{(1-r)(1+m r)}
$$

Consequently

$$
\mathrm{re} \frac{z f^{\prime}(z)}{f(z)} \geqslant \frac{q(r)}{\left(1-r^{2}\right)(1+m r)}
$$

where

$$
q(r) \equiv m r^{3}-3 m r^{2}-3 r+1 \text {. }
$$

The denominator of the expression on the right-hand side of inequality (42) is positive for $0 \leqslant r<1, q(0)=1>0, q(1)=-2(1+m)$ $<0$. Thus the smallest positive root $r_{0}$ of the equation $q(r)=0$ lies between 0 and 1.

Thus the inequality

$$
\mathrm{re} \frac{z f^{\prime}(z)}{f(z)}>0
$$

is valid for $r=|z|<r_{0}$. Hence the radius of starlikeness $R_{s}$ for $U(M)$ is not less than $r_{0}$. 
Equality in (41) holds for $z=-i r, \alpha=\frac{\pi}{2}, \beta=0, P(z)=\frac{1-i z}{1+i m z}$ and

$$
f^{*}(z)=\frac{z(1-i z)}{(1+i z)^{2}(1+i m z)} .
$$

Moreover, it can easily be verified that $f^{*}(z) \in U(M)$. Since

we get

$$
\frac{z f^{* \prime}(z)}{f^{*}(z)}=\frac{-i m z^{3}+3 m z^{2}-3 i z+1}{(1+i z)^{4}(1+i m z)^{2}}
$$

$$
\text { re } \frac{z f^{* \prime}(z)}{f^{*}(z)}=0
$$

for $z=-i r_{0}$. Thus the function $f^{*}(z)$ is not starlike in the disk $|z|<r$ for $r>r_{0}$. Hence, by $R_{s} \geqslant r_{0}$ we obtain $R_{s}=r_{0}$.

Now it remains to choose the smallest positive root $r_{0}$ of the equation

$$
m r^{3}-3 m r^{2}-3 r+1=0 .
$$

If $m \neq 0$, then (43) is cubic. Its discriminant is $-108 m(m+1)^{2}<0$. Consequently (43) has there distinct real roots for each $m \neq 0$; the smallest non-negative root is given by (39). In the limit case $m=1$ we obtain $\varrho=2-\sqrt{3}$. If $m=0$, then (43) is linear and we obtain $\varrho=\frac{1}{3}$.

6. An estimate of the radius of convexity for the family $C(M)$.

THEOREM 6. The radius of convexity $\varrho$ of the family $C(M)$ is not less, than the smallest positive root of the equation

$$
q(r) \equiv m r^{4}-2 m r^{3}-(1+m) r^{2}-2 r+1=0 .
$$

Proof. If $f(z) \epsilon C(M)$, then

$$
\left(1-z^{2}\right) f^{\prime}(z)=P(z)
$$

for some function $P(z) \in \mathscr{P}(M)$ with real coefficients. Differentiating (45) we obtain

$$
\left(1-z^{2}\right) f^{\prime \prime}(z)-2 z f^{\prime}(z)=P^{\prime}(z) .
$$

An easy calculation yields

$$
1+\frac{z f^{\prime \prime}(z)}{f^{\prime}(z)}=\frac{1+z^{2}}{1-z^{2}}+\frac{z P^{\prime}(z)}{P(z)}
$$

Thus

$$
\mathrm{re}\left(1+\frac{z f^{\prime \prime}(z)}{f^{\prime}(z)}\right) \geqslant \min _{|z|-r} \frac{1+z^{2}}{1-z^{2}}+\min _{\substack{|z|-r \\ P(z) \cdot \sigma(M)}} \mathrm{re} \frac{z P^{\prime}(z)}{P(z)} .
$$


Using (19) we obtain

$$
\mathrm{re}\left(1+\frac{z f^{\prime \prime}(z)}{f^{\prime}(z)}\right) \geqslant \frac{1-r^{2}}{1+r^{2}}-\frac{(1+m) r}{(1-r)(1+m r)}=\frac{q(r)}{(1-r)\left(1+r^{2}\right)(1+m r)}
$$

The denominator of the expression on the right-hand side of inequality (47) is positive for $0 \leqslant r<1, q(0)=1$ and $q(1)=-2(1+m)<0$. Thus the smallest positive root $r_{0}$ of equation (44) lies between 0 and 1 .

Therefore the inequality

$$
\mathrm{re}\left(1+\frac{z f^{\prime \prime}(z)}{f^{\prime}(z)}\right)>0
$$

is valid for $r=|z|<r_{0}$. Hence the radius of convexity for $C(M)$ is not less than $r_{0}$. The theorem is proved.

In the limit case $m=1$ we obtain a result of Libera [2].

In the case $m=0$ we have $\varrho \geqslant \sqrt{2}-1$.

CoROLLARY. The radius of starlikeness for the family $T(M)$ is not less than the smallest positive root of equation (44).

In fact, if $f(z) \in T(M)$, then

$$
\frac{1-z^{2}}{z} f(z)=P(z)
$$

for some function $P(z) \in \mathscr{P}(M)$ with real coefficients. Therefore

$$
\text { re } \frac{z f^{\prime}(z)}{f(z)}=\mathrm{re} \frac{1+z^{2}}{1-z^{2}}+\mathrm{re} \frac{z P^{\prime}(z)}{P(z)}
$$

(comp. (46)) and, consequently, we obtain the assertion.

7. The estimations of $|f(z)|,\left|f^{\prime}(z)\right|$ in $S^{*}(M)$.

Theorem 7. If $f(z) \in S^{*}(M)$, then for $|z|=r, 0 \leqslant r<1$

$$
\begin{gathered}
\frac{r}{(1+m r)^{(1+m) / m}} \leqslant|f(z)| \leqslant \frac{r}{(1-m r)^{(1+m) / m}} \quad \text { for } m \neq 0, \\
r \cdot e^{-r} \leqslant|f(z)| \leqslant r \cdot e^{r} \quad \text { for } m=0, \\
\frac{1-r}{(1+m r)^{(1+2 m) / m}} \leqslant\left|f^{\prime}(z)\right| \leqslant \frac{1+r}{(1-m r)^{(1+2 m) / m}} \quad \text { for } m \neq 0
\end{gathered}
$$

and

$$
(1-r) e^{-r} \leqslant\left|f^{\prime}(z)\right| \leqslant(1+r) e^{r} \quad \text { for } m=0 .
$$

These bounds are sharp, being attained by functions of form (34) and (35), respectively. 
Proof. Since

$$
\mathrm{r} \theta \frac{z f^{\prime \prime}(z)}{f^{\prime}(z)}=r \frac{\partial}{\partial r} \log \left|f^{\prime}(z)\right|, \quad|z|=r,
$$

and using (33) we obtain

$$
\frac{\partial}{\partial r} \log \left|f^{\prime}(z)\right| \geqslant \frac{(1+m)(r-2)}{(1-r)(1+m r)}
$$

Integrating both sides of this inequality from 0 to $r$, we obtain

and

$$
\left|f^{\prime}(z)\right| \geqslant \frac{1-r}{(1+m r)^{(1+2 m) / m}}, \quad \text { if } m \neq 0,
$$

$$
\left|f^{\prime}(z)\right| \geqslant(1-r) e^{-r}, \quad \text { if } m=0,
$$

i.e. the bound of the left of (50) and (51).

Let $z_{0},\left|z_{0}\right|=r$ this value of $z$, for which $\left|f\left(z_{0}\right)\right| \leqslant|f(z)|$, for all $z$, $|z|=r$. If $\Gamma$ is the pre-image of the segment $\left\langle 0, f\left(z_{0}\right)\right\rangle$, then

$$
\left|f\left(z_{0}\right)\right|=\int_{\Gamma}\left|f^{\prime}(z)\right||d z| \geqslant \int_{\Gamma}\left|f^{\prime}(z)\right| d r \geqslant \int_{0}^{r} \frac{1-r}{(1+m r)^{(1+2 m) / m}} d r, \quad \text { if } m \neq 0
$$

and

$$
\left|f\left(z_{0}\right)\right| \geqslant \int_{0}^{r}(1-r) e^{-r} d r, \quad \text { if } m=0 .
$$

From which we get the bounds of the left of (48) and (49). Since $f(z) \in S^{*}(M)$, then

$$
f(z)=z \exp \left(\int_{0}^{z} \frac{P(\zeta)-1}{\zeta} d \zeta\right), \quad P(z) \in \mathscr{P}(M) .
$$

Therefore

$$
|f(z)|=|z|\left(\mathrm{re} \int_{0}^{z} \frac{P(\zeta)-1}{\zeta} d \zeta\right)
$$

Substituing $\zeta=z t$, we obtain

$$
|f(z)|=|z|\left(\mathrm{re} \int_{0}^{1} \frac{P(z t)-1}{t} d t\right) .
$$

Hence

$$
|f(z)| \leqslant|z| \exp \left(\int_{0}^{1} \max _{|z t|=r t}\left(\mathrm{re}-\frac{P(z t)-1}{t}\right) d t\right)
$$


Because

$$
\max _{|z t|=r t} \mathrm{re} \frac{P(z t)-1}{t}=\frac{(1+m) r}{1-m r t}
$$

then, after integration, we obtain the upper bounds in (48) and (49). Similarly, we obtain the bounds of the right of (50) and (51), which ends the proof.

8. The estimations of the coefficients in $S^{*}(M)$ and $\mathscr{P}(M)$.

THEOREM 8. If $f(z) \in S^{*}(M)$, then

$$
\left|a_{n}\right| \leqslant \frac{1}{(n-1) !} \prod_{k=1}^{n-1}\left(1+k\left(1-\frac{1}{M}\right)\right), \quad n=2, \ldots, N
$$

and

$$
\left|a_{n}\right| \leqslant \frac{1}{(n-1)(N-2) !} \prod_{k=1}^{N-1}\left(1+k\left(1-\frac{1}{M}\right)\right), \quad n=N+1, N+2, \ldots
$$

where $N \in[2 M, 1+2 M)$, is natural.

Estimate (52) is sharp, equality holds for functions (34) and (35), respectively.

Proof. If $f(z) \in S^{*}(M)$, then

$$
\frac{z f^{\prime}(z)}{f(z)}=\frac{1+\omega(z)}{1-m \omega(z)}, \quad m=1-\frac{1}{M}
$$

for some function $\omega(z) \epsilon \Omega$ (comp. (6)).

From (53) it follows that

whence

$$
z f^{\prime}(z)-f(z)=\left(m z f^{\prime}(z)+f(z)\right) \omega(z),
$$

$$
\sum_{k=1}^{\infty}(k-1) a_{k} z^{k}=\omega(z) \sum_{k=1}^{\infty}(1+m k) a_{k} z^{k} \quad\left(a_{1}=1\right) .
$$

Equating the coefficient at $z^{2}$ on both sides of (54) we obtain $a_{2}$ $=(1+m) \omega^{\prime}(0)$. Since $\left|\omega^{\prime}(0)\right| \leqslant 1$, we obtain

$$
\left|a_{2}\right| \leqslant 1+m \text {. }
$$

Thus, estimate (52) holds for $n=2$.

Suppose $n>2$. We rewrite (54) as follows:

$$
\sum_{k=2}^{n}(k-1) a_{k} z^{k}+\sum_{k=n+1}^{\infty} c_{k} z^{k}=\omega(z) \sum_{k=1}^{n-1}(1+m k) a_{k} z^{k}
$$

where the sum $\sum_{k=n+1}^{\infty} c_{k} z^{k}$ is convergent in $K$. 
Let $z=r e^{i t}$, then since $|\omega(z)|<1$

$$
\begin{aligned}
& \sum_{k=2}^{n}(k-1)^{2}\left|a_{k}\right|^{2} r^{2 k}=-\frac{1}{2 \pi} \int_{0}^{2 \pi}\left|\sum_{k=2}^{n}(k-1) a_{k}\left(r e^{i t}\right)^{k}\right|^{2} d t \\
& \leqslant \frac{1}{2 \pi} \int_{0}^{2 \pi}\left|\sum_{k=1}^{n-1}(1+m k) a_{k}\left(r e^{i t}\right)^{k}\right|^{2} d t=\sum_{k=1}^{n-1}(1+m k)^{2}\left|a_{k}\right|^{2} r^{2 k}
\end{aligned}
$$

Passing to the limit in (57) as $r \rightarrow 1$, we conclude that

Therefore

$$
\sum_{k=2}^{n}(k-1)^{2}\left|a_{k}\right|^{2} \leqslant \sum_{k=1}^{n-1}(1+m k)^{2}\left|a_{k}\right|^{2}
$$

$$
(n-1)^{2}\left|a_{n}\right|^{2} \leqslant(1+m)^{2}+\sum_{k=2}^{n-1}\left[(1+m k)^{2}-(k-1)^{2}\right]\left|a_{k}\right|^{2} .
$$

We observe that $(1+m(n-1))^{2}-(n-2)^{2}>0$, if and only if $n \leqslant N$. By an inductive argument we obtain estimates (52) and (52').

The proof of this theorem is based on a method introduced by Clunie [1].

COROLLARY. The values assumed by $f(z)$ in $K$ include the disk

$$
|w|<\frac{1}{4-1 / m} \text {. }
$$

In fact, let $f(z)$ be in $S^{*}(M)$ and let $w$ be any number such that $f(z) \neq w$ for $z$ in $K$. Then the function

$$
g(z)=\frac{f(z)}{1-\frac{f(z)}{w}}=z+\left(a_{2}+\frac{1}{w}\right) z^{2}+\ldots
$$

belongs to $S$. Hence $\left|a_{2}+1 / w\right| \leqslant 2$. From which we obtain (58).

THeOREM 9. If $P(z) \in \mathscr{P}(M)$, then

$$
\left|b_{n}\right| \leqslant 2-\frac{1}{M}, \quad n=1,2,3, \ldots
$$

The bound is sharp, being attained if and only if

$$
P(z)=\frac{1+\varepsilon z^{n}}{1-\varepsilon\left(1-\frac{1}{M}\right) z^{n}}, \quad|\varepsilon|=1
$$

Proof. If $P(z) \in \mathscr{P}(M)$, then

$$
P(z)=\frac{1+\omega(z)}{1-m \omega(z)}
$$

for some function $\omega(z) \in \Omega$. 
From (61) it follows that

$$
P(z)-1=(1+m P(z)) \omega(z) .
$$

Let

$$
\omega(z)=c_{1} z+c_{2} z^{2}+\ldots+c_{n} z^{n}+\ldots
$$

Applying the metod of Clunie we obtain finally the inequality

$$
\left|b_{n}\right|^{2} \leqslant(1+m)^{2}+\left(m^{2}-1\right) \sum_{k=1}^{n-1}\left|b_{k}\right|^{2} .
$$

Since $m^{2}-1 \leqslant 0$,

$$
\left|b_{n}\right| \leqslant 1+m, \quad n=1,2,3, \ldots,
$$

i.e. we obtain estimation (59). Equality holds for function (60).

If $\left|b_{n}\right|=1+m, n=1,2,3, \ldots$, then from (63) we conclude that $b_{k}=0$ for $k=1,2, \ldots, n-1$. Therefore, from (62) we obtain $\left|c_{n}\right|=1$ and $c_{k}=0$ for $k \neq n$. Thus, the function $f(z)$ for which $\left|b_{n}\right|=1+m$ is of form $(60)$.

Corollary. The values assumed by $f(z)$ of $R(M)$ in $K$ include the disk

$$
|w|<\frac{2}{6-1 / M}
$$

\section{References}

[1] J. Clunie, On meromorphic schlicht functions, Journ. London Math. Soc. 34 (1859), pp. $215-216$.

[2] R. J. Libera, Some radius of convexity problems, Duke Math. J. 31 (1964), pp. $143-157$.

[3] Z. Nehari, Oonformal mapping, New York 1952.

[4] R. Nevanlinna, Über dio schlichten Abbildungen des Einheitskreises, Översikt av Finska Vetenskaps-Societentes Förhandlinger, T. 62 (1919-1920), Avd. A, N. 07.

[5] K. Noshiro, On the theory of schlicht functions, Journal of the Faculty of Science, Sapporo, vol. 2 (1934-1935), pp. $129-155$.

[6] M. S. Robertson, Analytio funotions starlike in one direction, Amer. J. of Math. 58 (1936), pp. $465-472$.

[7] - On the theory of schlicht functions, Ann. of Math. 37 (1936), pp. 169-185.

[8] - Variational methods for functions with positive real part, Trans. Amer. Math. Soc. 102 (1962), pp. $169-185$.

[9] W. Rogosinski, tber positive harmonische Entwicklungen und typischreelle Potenzroichen, Math. Z. 35 (1932), pp. 93-121. 